\title{
COPD as a disease of children: hype or hope for better understanding?
}

\section{David M Mannino}

"Train up a child in the way he should go: and when he is old, he will not depart from it." Proverbs 22:6

I have three children, two of whom are adults, and the third of whom is now taller than me. These children, despite sharing similar genes, are all very different in their personalities, demeanours, likes and dislikes. I am convinced that almost all of the traits they now have as adults, or near adults, are pretty much what they emerged from the womb with. Other parents I have discussed this observation with have similar stories-it may be that most of what affects us in adulthood has been established before we start grade school.

Almost all of the epidemiological studies of chronic obstructive pulmonary disease (COPD) have focused on risk factors in adulthood such as cigarette smoking, occupational exposures, exposures to biomass smoke and respiratory infections. ${ }^{1-4}$ COPD is clearly a disease that manifests itself in adulthood with a peak onset of incidence in the fifth to sixth decades of life. ${ }^{5}$ The paper in the current issue of Thorax by Svanes et al ${ }^{6}$ explores a different possibility-that COPD is a disease of childhood that becomes manifest in adults (see page 14). If this observation is correct, it may require us to reconsider some of our longheld assumptions on COPD.

According to the analysis by Svanes et $a l,{ }^{6}$ combining paediatric risk factors (such as a personal history of asthma, a parental history of asthma or early respiratory infections) with early exposure to tobacco smoke resulted in a risk that rivalled or exceeded that seen from the traditional COPD risk factor of cigarette smoking. The strongest risk factor was a diagnosis of "asthma" as a child, which was associated with a 10-fold higher risk of

\footnotetext{
Correspondence to: Dr D M Mannino, Department of Preventive Medicine and Environmental Health, University of Kentucky College of Public Health, 121 Washington Avenue, Lexington, KY 40536, USA; dmannino@uky.edu
}

stage 2 COPD in men and a 4-fold higher risk of stage 2 COPD in women.

The possibility that COPD starts in childhood is certainly provocative and has been the focus of several recent reviews or editorials. ${ }^{78}$ The argument that this is "hype" looks at 50 years worth of epidemiological and clinical work demonstrating that cigarette smoking is-at least in the developed world-the primary risk factor for COPD and that, in the absence of smoking, this disease would be greatly diminished. The supporters of this viewpoint might also say that focusing on anything other than tobacco smoking is a poor use of resources for a disease that is largely preventable. There has been sentiment in some circles to actually include cigarette smoking in the definition of $\mathrm{COPD}$, saying that disease occurring in never smokers is, by definition, something other than COPD. ${ }^{9}$

The alternative argument that childhood origins of COPD provide "hope for better understanding" is based on more recent data pointing to a substantial burden of COPD among never smokers and evidence based on cohorts of people followed since childhood. While cigarette smoking remains an undeniable risk factor, it is clear that not all smokers have the same risk of developing COPD and its complications.

Several possibilities may explain why we are now seeing evidence supporting the childhood origins of COPD. The first may be somewhat definitional. The increased willingness to accept impaired lung function as "confirmatory" of COPD is a recent change. While the precise definition may be controversial and well beyond the scope of this editorial, the idea that the presence of "abnormal lung function" is sufficient to diagnose COPD dramatically altered our epidemiological impression of disease burden, including the concept of "undiagnosed disease". 10-12 Another possibility is the increasing recognition that both asthma ${ }^{13}$ and COPD $^{14}$ have multiple phenotypes. Some asthma phenotypes in children do look like COPD in that they seem to be related to "irritant" exposure and show some evidence of impaired lung function. ${ }^{15}$ The improved treatment and survival of premature infants and their resulting chronic lung disease of prematurity is thought to be a potential risk factor for chronic pulmonary disease in adulthood. ${ }^{16}$ While this is an extreme example, it seems entirely feasible that less severe respiratory insults, especially when combined together, might result in respiratory diseases, including COPD, in adulthood.

So does early "training" of the respiratory system, paralleling the words of the proverb above, result in a tendency towards respiratory health or respiratory disease in adulthood? This certainly makes sense, and some recent data support the finding that measures of lung function at a mean age of 2 months track into young adulthood. ${ }^{17}$ Does this change our approach towards known risk factors such as cigarette smoking or occupational dust exposures? Of course not. While I believe adults entering adulthood with poor lung function are probably at an increased risk for developing COPD, there is no reason to believe that young adults with normal or even supernormal lung function are protected from COPD development. My hope is that a better understanding of the early life factors that increase the risk of disease might lead to better early detection and intervention efforts.

\section{Competing interests: None.}

Provenance and peer review: Commissioned; not externally peer reviewed.

Thorax 2010;65:1-2. doi:10.1136/thx.2009.118778

\section{REFERENCES}

1. Mannino DM, Buist AS. Global burden of COPD: risk factors, prevalence, and future trends. Lancet 2007; 370:765-73.

2. Liu S, Zhou Y, Wang $X$, et al. Biomass fuels are the probable risk factor for chronic obstructive pulmonary disease in rural South China. Thorax 2007;62:889-97.

3. Buist AS, McBurnie MA, Vollmer WM, et al. International variation in the prevalence of COPD (the BOLD Study): a population-based prevalence study. Lancet 2007; 370:741-50.

4. Blanc PD, Menezes AM, Plana E, et al. Occupational exposures and COPD: an ecological analysis of international data. Eur Respir J 2009;33:298-304.

5. Mannino DM, Watt G, Hole D, et al. The natura history of chronic obstructive pulmonary disease. Eur Respir J 2006;27:627-43.

6. Svanes C, Sunyer J, Plana E, et al. Early life origins of chronic obstructive pulmonary disease. Thorax 2009;65:14-20.

7. Martinez FD. The origins of asthma and chronic obstructive pulmonary disease in early life. Proc Am Thorac Soc 2009;6:272-7.

8. Bush A. COPD: a pediatric disease. COPD 2008;5:53-67.

9. Miravitlles M, Morera J. It's time for an aetiology based definition of chronic obstructive pulmonary disease. Respirology 2007;12:317-9. 
10. Mannino DM, Gagnon RC, Petty TL, et al. Obstructive lung disease and low lung function in adults in the United States: data from the National Health and Nutrition Examination Survey, 1988-1994. Arch Intern Med 2000;160:1683-9.

11. Shahab L, Jarvis MJ, Britton J, et al. Chronic obstructive pulmonary disease prevalence, diagnosis and relation to tobacco dependence in a nationally representative population sample. Thorax 2006;61:1043-7.
12. Kim SJ, Suk MH, Choi HM, et al. The local prevalence of COPD by post-bronchodilator GOLD criteria in Korea. Int J Tuberc Lung Dis 2006;10:1393-8.

13. Wenzel SE. Asthma: defining of the persistent adult phenotypes. Lancet 2006;368: 804-13.

14. Friedlander AL, Lynch D, Dyar LA, et al. Phenotypes of chronic obstructive pulmonary disease. COPD 2007; 4:355-84.
15. Kelley CF, Mannino DM, Homa DM, et al. Asthma phenotypes, risk factors, and measures of severity in a national sample of US children. Pediatrics 2005:115:726-31.

16. Baraldi E, Filippone M. Chronic lung disease after premature birth. N Engl J Med 2007;357:1946-55.

17. Stern DA, Morgan WJ, Wright AL, et al. Poor airway function in early infancy and lung function by age 22 years: a non-selective longitudinal cohort study. Lancet 2007;370:758-64.

\section{A Bill of "Rights" for patients with COPD: the "right" therapy for the "right" patient at the "right" time}

\section{Linda Nici}

Chronic obstructive pulmonary disease (COPD) is a major cause of morbidity and mortality and poses significant burdens on patients, their families and the healthcare system. It is currently the fourth leading cause of death in the USA and is projected to rank fifth in burden of disease globally by 2020. ${ }^{1}$ Pulmonary rehabilitation (PR) for COPD is an effective therapeutic intervention that reduces breathlessness, increases exercise capacity and improves health-related quality of life. $^{23}$ Emerging evidence also supports its effectiveness in reducing healthcare utilisation, decreasing frequency and severity of acute exacerbations and improving survival. ${ }^{4-7}$

Despite the robust evidence on the effectiveness of this intervention, the availability of PR remains dismal worldwide, with various estimates suggesting $1-14 \%$ of patients with COPD are actually referred for the service..$^{-10}$ This partly has to do with the fact that PR is most often delivered as an outpatient and hospital-based programme. If PR could be delivered effectively in the home setting, this could vastly increase the number of patients receiving this important therapy. Simply put, the right therapy for the right patient at the right time.

Few studies have looked at the efficacy of home-based rehabilitation, but the

Correspondence to: Dr L Nici, Department of Medicine, The Warren Alpert Medical School of Brown University, Associate Chief, Pulmonary and Critical Care Section, Providence Veterans Affairs Medical Center, Providence, RI 02908, USA; linda_nici@brown.edu programmes vary widely, making comparisons difficult. One study from the Netherlands looked at a 12-week programme using community-based healthcare personnel and visits to local physiotherapists. ${ }^{11}$ While the study did demonstrate feasibility of the programme, high exercise compliance as assessed by patient diaries and some improvement of exercise tolerance, patients attended supervised exercise training twice weekly. This is very similar to traditional outpatient programmes in the USA.

In contrast, a study from Spain looked at the effects of an 8-week supervised versus self-monitored exercise training programme. ${ }^{12}$ Both groups improved exercise tolerance, but the magnitude of physiological improvement was greater in supervised patients. This study, while truly home-based, had no other components of PR so the results are difficult to compare with traditional comprehensive programmes.

A more recent study from Canada delivered a self-management education programme prior to either a standard outpatient hospital-based exercise programme or a home-based self-monitored exercise programme. ${ }^{13}$ This study showed similar improvements in dyspnoea and health status measurements but, while closer to a comprehensive home-based programme than the two previously mentioned studies, patients received the education component outside of the home; therefore the results reflect effectiveness of home-based exercise.

Clearly, more work is needed to develop and evaluate PR programmes that can deliver the essential components of exercise and education, yet allow for easy accessibility of this service in the home setting where compliance and long-term adherence may be higher.

Another consequence of the lack of availability of $\mathrm{PR}$ is that it is often reserved as a "last ditch" effort in patients with severe and very severe COPD. The good news is that these severely impaired patients derive substantial benefit from PR. ${ }^{14}$ However, long-term maintenance of benefits can be hampered by increased frailty, making adherence to healthy behaviours including regular exercise increasingly difficult.

This provides yet another challenge to the PR specialist-to provide and encourage $P R$ in patients with milder disease. If patients are exposed to therapeutic interventions that promote self-efficacy early in the course of a chronic disease, then there may be a real and measurable impact on disease progression. Strategies employed during comprehensive PR such as smoking cessation, exercise training and teaching self-care could be delivered individually or as a package over the life time of the patient with COPD. Again, the right therapy for the right patient at the right time.

In addition to this theoretical argument to provide PR through a continuum of care to optimise chronic disease management, there is another strong argument to provide PR to patients with milder disease. There are significant systemic effects of COPD even in patients who are deemed mild or moderate by forced expiratory volume in $1 \mathrm{~s}\left(\mathrm{FEV}_{1}\right)$ staging criteria. Effects such as skeletal muscle dysfunction, body composition abnormalities and poor self-management skills are targeted by the components of PR. Unfortunately, the need for PR is often overlooked when patients are assessed by physiological parameters alone.

Impaired exercise capacity has been shown to be a significant determinant of disease burden, not only in patients with severe and very severe COPD but also in those with moderate disease..$^{15}$ Differences in body composition can already be seen 